\title{
Upcoming Events in Pediatric Cardiology
}

April 3-5, 2011

New Orleans, Louisiana, USA

American College of Cardiology, Annual Scientific Session

http://www.acc.org

May 7-11, 2011

Philadelphia, Pennsylvania, USA

American Association of Thoracic Surgery, Annual Meeting

http://www.aats.org

July 24-27, 2011

Pediatric Interventional Cardiology Symposium-AICS

Boston, Massacheussets, USA

http://www.picsymposium.com

October 22-27, 2011

Honolulu, Hawaii, USA

Annual Scientific Assembly, American College of Chest Physicians

Telephone: 847/498-1400

http://www.chestnet.org/CHEST
November 13-15, 2011

Orlando, Florida, USA

Scientific Session, American Heart Association

http://scientificsessions.americanheart.org

January 30-February 1, 2012

Fort Lauderdale, Florida, USA

Annual Meeting of the Society of Thoracic Surgery http://www.sts.org

February 22-26, 2012

Orlando, Florida, USA

Update on Pediatric and Congenital Cardiovascular Disease

http://heart.chop.edu/cardiology 2012 